Ido G. Bikker

Steffen Leonhardt

Jan Bakker

Diederik Gommers

\section{Lung volume calculated from electrical impedance tomography in ICU patients at different PEEP levels}

Received: 5 December 2008

Accepted: 5 May 2009

Published online: 10 June 2009

(C) The Author(s) 2009. This article is published with open access at Springerlink.com

Electronic supplementary material The online version of this article (doi:10.1007/s00134-009-1512-6) contains supplementary material, which is available to authorized users.
I. G. Bikker · J. Bakker · D. Gommers ( Department of Intensive Care Medicine, Room H602, Erasmus MC, Postbox 2040, 3000 CA Rotterdam, The Netherlands e-mail: d.gommers@erasmusmc.nl Tel.: +31-10-7040704

Fax: +31-10-7032874

\section{S. Leonhardt}

Philips Chair for Medical Information

Technology, Helmholtz-Institute

for Biomedical Engineering,

RWTH Aachen University,

Aachen, Germany
Abstract Purpose: To study and compare the relationship between end-expiratory lung volume (EELV) and changes in end-expiratory lung impedance (EELI) measured with electrical impedance tomography (EIT) at the basal part of the lung at different PEEP levels in a mixed ICU population. Methods: End-expiratory lung volume, EELI and tidal impedance variation were determined at four PEEP levels (15-10-5-0 cm $\mathrm{H}_{2} \mathrm{O}$ ) in 25 ventilated ICU patients. The tidal impedance variation and tidal volume at $5 \mathrm{~cm} \mathrm{H}_{2} \mathrm{O}$ PEEP were used to calculate change in impedance per ml; this ratio was then used to calculate change in lung volume from change in EELI. To evaluate repeatability, EELV was measured in quadruplicate in five additional patients. Results: There was a significant but relatively low correlation $\left(r=0.79 ; R^{2}=0.62\right)$ and moderate agreement (bias $194 \mathrm{ml}$, SD $323 \mathrm{ml}$ ) between $\triangle E E L V$ and change in lung volume calculated from the $\Delta E E L I$. The ratio of tidal impedance variation and tidal volume differed between patients and also varied at different PEEP levels. Good agreement was found between repeated EELV measurements and washin/washout of a simulated nitrogen washout technique. Conclusion: During a PEEP trial, the assumption of a linear relationship between change in global tidal impedance and tidal volume cannot be used to calculate EELV when impedance is measured at only one thoracic level just above the diaphragm.

Keywords EIT - Functional residual capacity · Humans - Critical care ·

Positive-pressure respiration .

Lung volume measurements

\begin{tabular}{|c|c|}
\hline \multicolumn{2}{|c|}{ Abbreviations } \\
\hline CT & Computed tomography \\
\hline EIT & $\begin{array}{l}\text { Electrical impedance } \\
\text { tomography }\end{array}$ \\
\hline EELV & End-expiratory lung volume \\
\hline EELI & $\begin{array}{l}\text { End-expiratory lung } \\
\text { impedance }\end{array}$ \\
\hline ICU & Intensive care unit \\
\hline PEEP & $\begin{array}{l}\text { Positive end-expiratory } \\
\text { pressure }\end{array}$ \\
\hline
\end{tabular}

\section{Introduction}

Electrical impedance tomography (EIT) may be a promising new tool for bedside monitoring of regional lung ventilation and changes in end-expiratory lung volume (EELV) $[1,2]$.
EIT is a technique based on the injection of small currents and voltage measurements using electrodes on the skin surface generating cross-sectional images representing impedance change in a slice of the thorax. It is a radiation free, non-invasive and portable lung imaging technique. 
When there is a linear relationship between $\Delta$ impedance and $\Delta$ volume, EIT can be used to calculate dynamic EELV changes based on impedance changes. Adler et al. [3] demonstrated a linear increase in impedance in dogs when the lung was inflated with a calibrated syringe. Also, EELV measured by an open-circuit nitrogen washout maneuver increased nearly linearly with impedance change ( $n=10, R^{2}=0.95$ ) [4]. This principle was used during various procedures with rapidly changing lung volumes to calculate a dynamic EELV in the whole lung, calibrated with impedance change and tidal volumes at one thoracic level [5-7]. However, it remains unclear whether this principle is only valid for an open lung state and perhaps not for a diseased lung with recruitment/ derecruitment as a result of PEEP changes at the site of the EIT measurement. In ventilated critically ill patients, ventilation is not divided homogenously [8], and, besides change in air volume, other factors (e.g., cardiac oscillations and volume of the pulmonary vascular bed) may contribute to impedance change. During a PEEP trial, inter-electrode distance and chest geometry (EIT assumes a round chest) will be affected. Furthermore, EIT measures an eclipse with a central diameter of approximately 5$10 \mathrm{~cm}$. During an incremental PEEP trial, with increased lung volume, the lung regions move in the cranio-caudal axis, and the individual pixel of the EIT map may no longer be representative for the same lung region [9]. Because the electrical current flows on three-dimensional trajectories, it remains unclear how movements of inhomogenous lung tissue in the cranio-caudal axis affect the diagnostic value of EIT measurements in the supine patient.

Therefore, this study aimed to evaluate the relationship between measured EELV and changes in volume calculated from $\triangle E E L I$ measured at the basal part of the lung during a PEEP trial in a mixed ICU population.

\section{Materials and methods}

The study population consisted of a convenient sample of 30 mechanically ventilated patients. The study data were obtained during PEEP trials integrated in standard practice, with the approval and need for informed consent waived for routinely collected data by the local human investigations committee. For all patients, chest X-rays and CT scans (if available) were retrospectively evaluated and related to clinical history and data to divide the patients into four groups: (1) patients without acute respiratory failure (non-ARF; group N), (2) with respiratory failure due to primary lung disorders (group P), (3) patients with respiratory failure due to secondary lung disorders (group $S$ ) and (4) patients admitted to the ICU after major surgery (group MS). All patients were adequately sedated and had a regular breathing pattern. Patients with an air leak due to pneumothorax, severe airflow obstruction due to COPD (defined as forced expired volume in $1 \mathrm{~s}$ or vital capacity below predicted value minus 2 SD) and severe cardiovascular instability were excluded from the study.

In 25 patients impedance measurements were performed during 2 min with a silicone belt with 16 integrated electrocardiographic electrodes placed around the thoracic cage at the fifth or sixth intercostal space, connected with an EIT device (EIT evaluation kit 2, Dräger, Lubeck, Germany). EIT data were generated by application of a small alternating electrical current of $5 \mathrm{~mA}$ and $50 \mathrm{kHz}$. During the study period, patients were ventilated with an Engström Carestation ventilator (GE Healthcare, Madison, WI). EELV measurements were performed with the COVX module (GE Healthcare, Helsinki, Finland) integrated within the ventilator. Lung volumes were calculated based on a multibreath simulated nitrogen washout by measurement of oxygen and carbon dioxide. This COVX module has been extensively described by Olegard et al. [10] and recently validated in ICU patients by Chiumello et al. [11]. At baseline, patients were switched to the Engström ventilator and ventilated according to their original settings before any measurements were made. After a steady state of at least $20 \mathrm{~min}$, PEEP was increased to $15 \mathrm{~cm} \mathrm{H}_{2} \mathrm{O}$ without changing the other ventilator settings. After a steady state, EELV was measured twice (washout and washin). This was repeated at a PEEP of 10,5 and (if clinically acceptable) $0 \mathrm{~cm} \mathrm{H}_{2} \mathrm{O}$. Before each EIT and EELV measurement, hemodynamic and ventilatory parameters were recorded, and arterial blood gas analysis was performed (ABL 700, Radiometer, Copenhagen, Denmark) in order to calculate the $\mathrm{PaO}_{2} / \mathrm{FiO}_{2}$ ratio. Dynamic compliance was calculated by dividing expiratory tidal volume by the driving pressure.

In five additional patients quadruplicate EELV measurements were made at three PEEP levels $(15,10$ and $5 \mathrm{~cm} \mathrm{H}_{2} \mathrm{O}$ ) to evaluate the repeatability of the simulated nitrogen washout technique. PEEP was increased from the original settings to $15 \mathrm{~cm} \mathrm{H}_{2} \mathrm{O}$, and after a steady state of $20 \mathrm{~min}$ the EELV was measured in quadruplicate (4 washins and 4 washouts). This was repeated after a steady state of $15 \mathrm{~min}$ at 10 and $5 \mathrm{~cm} \mathrm{H}_{2} \mathrm{O}$.

\section{EIT analysis}

EIT data were stored and analyzed offline on a personal computer (Dell, P4, $2.4 \mathrm{GHz}$ ). Figure 1 presents a diagram of a representative patient. At each designated PEEP level, mean tidal impedance variation and mean EELI were calculated. The ratio between tidal impedance variation and tidal volume at $5 \mathrm{~cm} \mathrm{H}_{2} \mathrm{O}$ PEEP was used to calculate $\Delta$ volume from the EELI between the different PEEP levels. $\triangle$ Mean EELI between PEEP levels was divided by this ratio to calculate a $\Delta$ lung volume. $\Delta$ Lung volume was calculated between each PEEP level and the lowest used PEEP level (0 or $5 \mathrm{~cm} \mathrm{H}_{2} \mathrm{O}$ ). Distribution of tidal ventilation was 


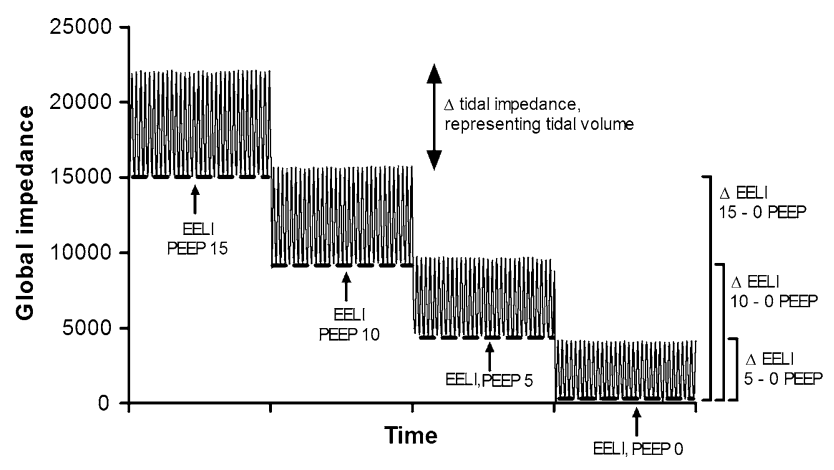

Fig. 1 Electrical impedance tomography (EIT) recording in a patient at different PEEP levels. $\triangle$ End-expiratory lung impedance (EELI) was calculated as the difference between EELI at each PEEP level and the lowest used PEEP level

expressed with the center of gravity index [12]. Change in global tidal impedance was divided into two equal regions of interest, dorsal and ventral, each accounting for $50 \%$ of the EIT image. The center of gravity was calculated by dividing the dorsal tidal impedance variation by the total tidal impedance variation.

\section{Statistical analysis}

Statistical analysis was performed with Graphpad software package (Graphpad software Inc. San Diego, CA). Results are expressed as mean $\pm \mathrm{SD}$ for normal distributed data and median + interquartile range (IQR) for not normally distributed data. The Shapiro-Wilk normality test was used to evaluate the distribution of all data. Changes in EELV measured with the nitrogen washout/in method and changes in EELV calculated from EELI were analyzed with linear regression, Spearman's correlation and a Bland-Altman analysis. Wilcoxon's matched-pairs test was used to analyze the tidal impedance variation and the tidal volume ratio.

The repeatability of the quadruplicate EELV measurements in five patients was evaluated with the variation coefficient (SD divided by the average). Bland-Altman analysis was used to analyze the agreement between washin/washout measurements in these five patients.

\section{Results}

This study examined the relation between EELV and EELI in 25 mechanically ventilated patients. Table 1 presents physiologic and demographic data on the study population.

Patients were retrospectively divided into four groups: group $\mathrm{N}(n=9)$ consisted of two patients with traumatic brain injury, five with cerebral vascular accidents and two with a postoperative condition after neurosurgery; group $\mathrm{P}(n=6)$ consisted solely of patients with pneumonia;
Table 1 Data on patient characteristics

\begin{tabular}{lll}
\hline & $\begin{array}{l}\text { EELI-EELV } \\
\text { relationship }\end{array}$ & $\begin{array}{l}\text { EELV } \\
\text { repeatability }\end{array}$ \\
\hline$n$ & 25 & 5 \\
Gender, female/total & $8 / 25$ & $4 / 5$ \\
Age (years) & $51(15)$ & $59(21)$ \\
Height $(\mathrm{cm})$ & $177(9)$ & $172(11)$ \\
Weight $(\mathrm{kg})$ & $76(12)$ & $74(26)$ \\
Time after intubation (hours) & $17.2(11.0)$ & $28.4(17.2)$ \\
Baseline $\mathrm{PEEP}_{\left(\mathrm{cm} \mathrm{H}_{2} \mathrm{O}\right)}$ & $7.3(2.7)$ & $8.6(2.2)$ \\
Baseline $\mathrm{PaO} \mathrm{F}_{2} / \mathrm{FiO} \mathrm{O}_{2}$ ratio & $344(533-123)$ & $245(360-145)$ \\
$\quad($ mmHg) (range) & & \\
Ventilation mode & 15 & 4 \\
Pressure control & 5 & 1 \\
Pressure support & 1 & \\
Volume control & 4 & \\
Pressure controlled- & & \\
$\quad$ volume guaranteed & & \\
\hline
\end{tabular}

Data are presented as mean (SD), unless stated otherwise $P E E P$ Positive end-expiratory pressure, EELI end-expiratory lung impedance, $E E L V$ end-expiratory lung volume

group S $(n=4)$ consisted of patients with abdominal sepsis; in group MS $(n=6)$ two patients were admitted to the ICU after liver transplantation, one after kidney transplantation, two after trans-thoracic esophagectomy and one patient after coronary bypass surgery.

Of the 25 patients, 20 were ventilated with pressure or volume-controlled ventilation and 5 patients with pressure support ventilation; all had a stable breathing pattern and stable EELI. Mean time after intubation was $17.2 \mathrm{~h}$. In total, 13 patients were measured at a PEEP of 15,10 and $5 \mathrm{~cm} \mathrm{H}_{2} \mathrm{O}$ and 12 patients at a PEEP of $15,10,5$ and $0 \mathrm{~cm} \mathrm{H}_{2} \mathrm{O}$. In five additional ICU patients we evaluated the repeatability of the simulated nitrogen washout technique; all patients tolerated this procedure well. Table 1 also presents data on the characteristics of this group.

The correlation between $\Delta$ lung volume measured with a multibreath simulated nitrogen washout technique and slung volume calculated from EELI is shown in Fig. A (see Electronic supplementary materials); although significant, the correlation was moderate $\left(r=0.79, R^{2}=\right.$ 0.62 ). To assess the difference between the two methods, a Bland-Altmann analysis was performed (Fig. 2). Calculating slung volume from EELI resulted in an overestimation compared with the multiple breath washout technique (bias $194 \mathrm{ml}$ ). Large differences in $\Delta$ lung volume were found between the two methods (SD $323 \mathrm{ml}$ ). To calculate $\Delta$ lung volume from EIT, the ratio or slope between tidal impedance variation and tidal volume at $5 \mathrm{~cm} \mathrm{H}_{2} \mathrm{O}$ was used. This ratio is shown in Fig. 3 for each group of patients at the PEEP levels applied. During the decremental PEEP trial, overall the decrease in the slope was significant $(15$ vs. 5: $P<0.001 ; 10$ vs. 5: $P<0.001 ; 0$ vs. $\left.5 \mathrm{~cm} \mathrm{H}_{2} \mathrm{O}: P=0.001\right)$. In all individual patient categories this ratio was different at 15 versus $5 \mathrm{~cm}$ $\mathrm{H}_{2} \mathrm{O}$ PEEP; between patients the slopes also differed. 


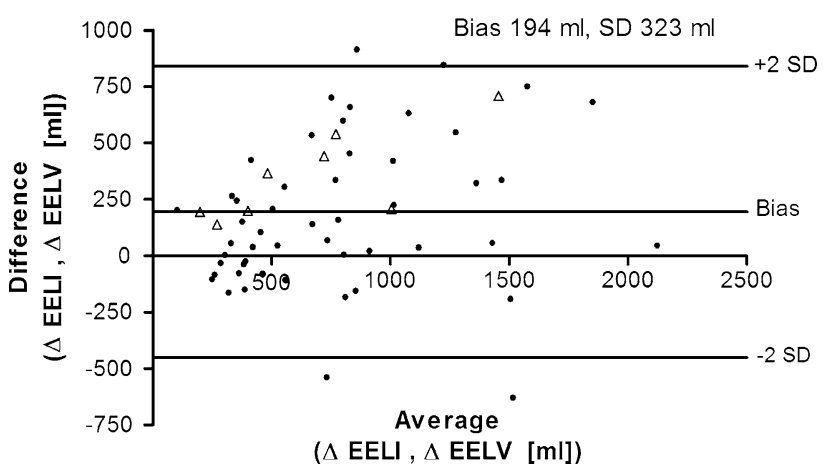

Fig. 2 Bland-Altman analysis. Comparison of $\Delta$ end-expiratory lung volume $(\triangle E E L V)$ measured with a multibreath nitrogen washout technique and $\Delta$ lung volume obtained from changes in end-expiratory lung impedance (EELI) calculated with the slope between tidal impedance variation and tidal volume at $5 \mathrm{~cm} \mathrm{H}_{2} \mathrm{O}$ PEEP. Open triangles represent patients on assisted spontaneous breathing, and black dots represent patients on controlled ventilation. The linear correlation is shown in Fig. A (see Electronic supplementary materials)

The right panel of Fig. 3 shows the dorsal to ventral center of gravity index of the tidal impedance variation for each patient group. In group $\mathrm{N}$ and group $\mathrm{MS}$ there was a significantly higher center of gravity at 15 and 10 compared to $5 \mathrm{~cm} \mathrm{H}_{2} \mathrm{O}$ PEEP, indicating increased tidal ventilation distribution in the dorsal, dependent lung areas.

Figure 4 shows arterial oxygenation and dynamic compliance for each patient group. No significant differences were found between the groups; however, this may be due to the small numbers of patients per group.

In the five patients with quadruplicate EELV measurements (used to evaluate repeatability of the simulated nitrogen washout technique), mean EELV was 2,232 (SD
1,468), 1,992 (SD 1,304) and 1,565 (SD 1,042) $\mathrm{ml}$ at 15, 10 and $5 \mathrm{~cm} \mathrm{H}_{2} \mathrm{O}$ PEEP, respectively. There was good agreement between the measurements with variation coefficients of 0.018 (range 0.012-0.027), 0.032 (0.018$0.050)$ and $0.039(0.005-0.101)$, and within subjects the SDs were 34 (range 11-52), 29 (10-48) and 23 (7-38) $\mathrm{ml}$ at 15,10 and $5 \mathrm{~cm} \mathrm{H}_{2} \mathrm{O}$ PEEP, respectively. Also, there was good agreement between the washin and washout measurements, bias $-0.76 \pm 3.22 \%$.

\section{Discussion}

This study assessed the relationship between $\Delta$ lung volume measured with a simulated nitrogen washout technique and $\Delta$ lung volume calculated from EIT in critically ill patients. Compared to the nitrogen washout technique, EIT overestimated $\Delta$ lung volume, and large overestimations or underestimations occurred in the individual patient. The ratio between tidal impedance variation and tidal volume decreased at lower PEEP levels, indicating a change in ventilation distribution at lower PEEP.

This study has some methodological considerations that need to be addressed. First, both techniques (EIT and the simulated nitrogen washout technique integrated into the Engström Carestation ventilator) are novel techniques with ongoing development. Second, Olegard et al. [10] investigated the precision of the simulated nitrogen washout technique and found a bias of $-9 \mathrm{ml}$ and limits of agreement of $356 \mathrm{ml}$ when performing repeated measurements with an $\mathrm{FiO}_{2}$ step of 0.1 and 0.3 ; this could in part explain the moderate correlation found in the present study.

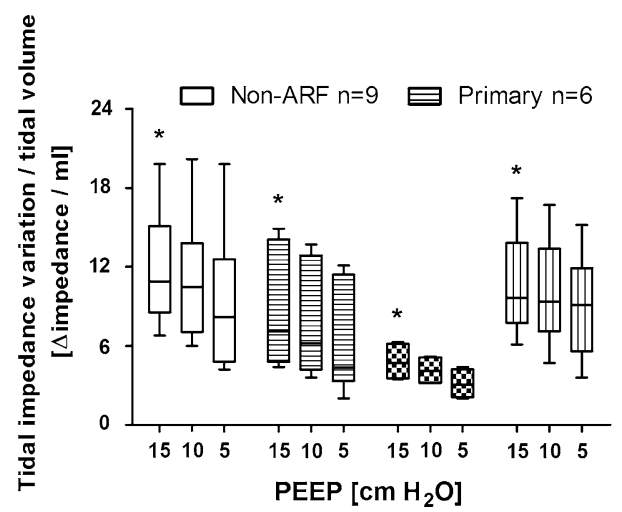

Fig. 3 Tidal impedance variation at the studied PEEP levels. Left panel shows the ratio of tidal impedance variation divided by tidal volume (impedance/ml). This ratio is the slope between tidal impedance variation and tidal volume. Right panel shows the center of gravity index. Global tidal impedance variation was divided in two equal regions of interest, dorsal and ventral, each accounting for $50 \%$ of the EIT image. The center of gravity was calculated by dividing the dorsal tidal impedance variation by the total tidal

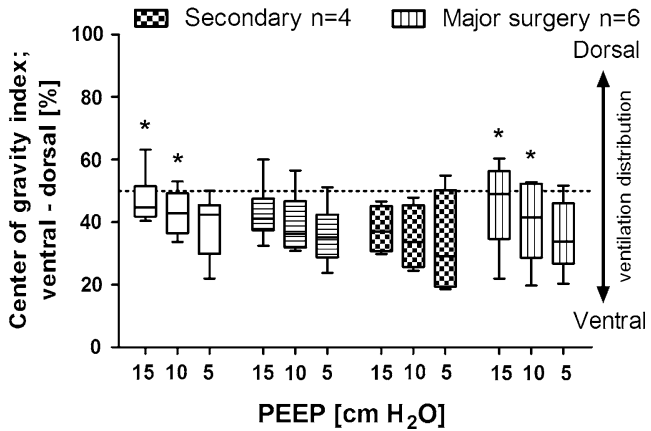

impedance variation. Patients are divided into groups according to the type of lung condition. Group $\mathrm{N}=$ non-ARF, group $\mathrm{P}=$ primary lung disorder, group $\mathrm{S}=$ secondary lung disorder, group $\mathrm{MS}=$ admitted to the ICU after major thoracic or abdominal surgery. Data are presented as box=and-whisker plot (min. $-25-50-$ 75\%-max.), asterisks indicate significance compared to $5 \mathrm{~cm} \mathrm{H}_{2} \mathrm{O}$ PEEP within each group, $P<0.05$ 


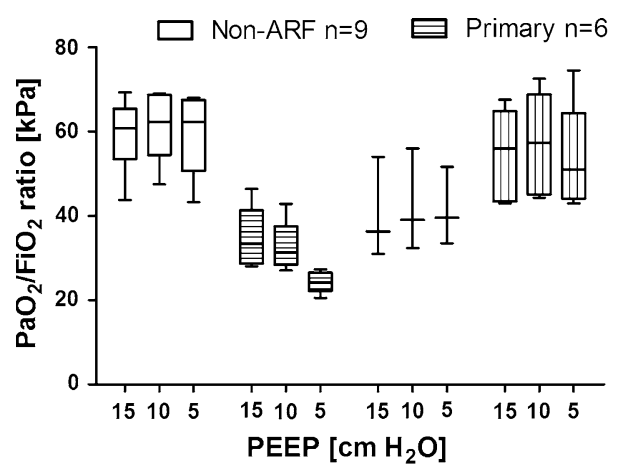

Fig. $4 \mathrm{PaO}_{2} / \mathrm{FiO}_{2}$ ratio (left) and dynamic compliance (right) at the PEEP levels examined in the four patient groups. Patients are divided into groups according to the type of lung condition. Group $\mathrm{N}=$ non-ARF, group $\mathrm{P}=$ primary lung disorder, group

However, the simulated nitrogen washout method did correlate well in a recent comparison with helium dilution and CT scanning [11]. Also, we evaluated the repeatability of the simulated nitrogen washout technique at 15,10 and $5 \mathrm{~cm} \mathrm{H}_{2} \mathrm{O}$ PEEP and found good agreement between the repeated measurements. Therefore, and based on the significantly lower ratios between tidal impedance variation and tidal volume during the decremental PEEP trial, we believe that the results also reflect lung physiology.

The results of the present study do not support the assumption of a purely linear relationship between $\Delta$ volume and $\Delta$ impedance in critically ill patients, especially as impedance was measured at only one thoracic level. Although we found a significant correlation, the level of agreement was poor. A linear relationship has been reported in different settings. For example, Erlandsson et al. [5] described good agreement between impedance tidal variation and tidal volume $\left(R^{2}=0.95\right)$ in morbidly obese patients undergoing surgery. The results of our study do not contradict these findings, because tidal volume is less likely to affect recruitment or derecruitment compared to PEEP change in the whole lung. In animal studies, good agreement was found between impedance and volume when inflating the lung with a calibrated syringe $[3,13]$. Hinz et al. [4] measured impedance and lung volume with an open circuit nitrogen washout technique in ten critically ill patients with acute respiratory failure. In their study, the patients were a heterogeneous group with different causes of respiratory failure, and the measured EELV ranged from 815 to $2,002 \mathrm{ml}$ at $0 \mathrm{~cm} \mathrm{H}_{2} \mathrm{O}$ PEEP. Agreement between change in EELV and change in EELI was good $\left(R^{2}=0.95\right)$. Surprisingly, the slope between $\Delta$ volume and $\Delta$ impedance was very similar for all their patients [4]. In our study, however, we found different slopes between tidal impedance variation and tidal volume for each patient (Fig. 3). This slope is dependent on the electrical conductivity of the measured tissue and may be influenced by edema and other lung pathology $[14,15]$. In the present study, the different slopes explain the moderate correlation,

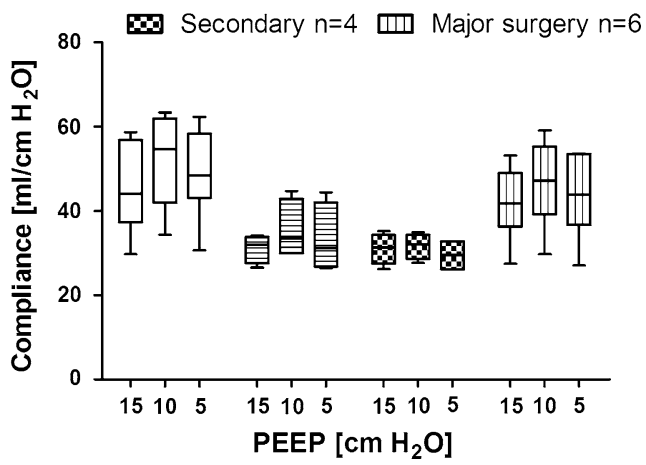

$\mathrm{S}=$ secondary lung disorder, group MS = admitted to the ICU after major thoracic or abdominal surgery. Data are presented as box-and-whisker plot (min.-25-50-75\%-max.)

because they indicate a change in the distribution of ventilation at different PEEP levels. With EIT, PEEP has been shown to shift ventilation in the frontal to dorsal direction [8]. This phenomenon was also present in the present study, indicated by the center of gravity index (Fig. 3). At higher PEEP levels, tidal ventilation distribution made a significant shift in groups $\mathrm{N}$ and MS to the dorsal parts of the lung, indicating decreased atelectasis. Also, with atelectasis in the dependent regions near the diaphragm, PEEP is likely to shift ventilation and air content in the cranial-to-caudal direction. Consequently, $\Delta$ volume calculated from $\Delta$ impedance measured at one thoracic level would not be representative for $\Delta$ volume in the entire lung. This explanation is supported by the difference in time between measurement and intubation, because lung recruitment with increased airway pressures is more effective at the start of mechanical ventilation. In the study of Hinz et al., patients were intubated on average $7 \pm 3$ days, whereas in the present study patients were ventilated only $17.2 \pm 11.0 \mathrm{~h}$ before measurement.

While the results of this study indicate that EIT measured at one basal thoracic level cannot predict lung volume changes in the entire lung, EIT has been shown to reliably assess ventilation distribution as compared with CT scanning. Victorino et al. [16] compared EIT with the same CT slice and showed good agreement between EIT images and dynamic CT scanning, good agreement in detection of right-left ventilation imbalance and relative ventilation distribution in layers of the thoracic section. Furthermore, in anesthetized pigs a good correlation was shown between the changes in lung air content determined by EIT and electron beam CT [17]. An experimental ALI study by Meier et al. [18] explored global and regional lung recruitment and lung collapse, and compared EIT with corresponding CT slices during an incremental and decremental PEEP trial; here, a strong correlation between regional volume changes was established. However, even in this latter study the intra-individual relationship between tidal volume and tidal impedance was much 
stronger than the overall correlation between all studied animals $(n=8)$. Thus, EIT has been shown to be very precise and reproducible, especially when looking at regional ventilation and not at overall lung volume [18].

In conclusion, the assumption of a strictly linear relationship between global tidal impedance variation and tidal volume cannot be used to calculate EELV based on $\triangle$ EELI during a PEEP trial when impedance is measured only at one thoracic level just above the diaphragm. This is particularly so when there is considerable change in the geometry of the electrical current conduction pathways such as, for example, in the case of recruitment and derecruitment. In this case, the relationship may become nonlinear. Thus, there might be a different slope between tidal impedance variation and tidal volume in different patients. Nevertheless, this intra-individual slope can be used to monitor regional ventilation distribution to assess the effect of ventilator settings.

Acknowledgments The authors thank Laraine Visser-Isles for English language editing. The EIT equipment was supplied by Dräger Medical AG, Lübeck, Germany. The Department of Intensive Care Medicine, Erasmus MC, received an unrestricted grant from GE Healthcare, Hoevelaken, The Netherlands.

Open Access This article is distributed under the terms of the Creative Commons Attribution Noncommercial License which permits any noncommercial use, distribution, and reproduction in any medium, provided the original author(s) and source are credited.

\section{References}

1. Brunow DC, Fonseca MC, Johnston C (2007) Electric impedance tomography, the final frontier is close: the bedside reality. Crit Care Med 35:1996-1997

2. Putensen C, Wrigge H, Zinserling J (2007) Electrical impedance tomography guided ventilation therapy. Curr Opin Crit Care 13:344-350

3. Adler A, Amyot R, Guardo R, Bates JH, Berthiaume Y (1997) Monitoring changes in lung air and liquid volumes with electrical impedance tomography. J Appl Physiol 83:1762-1767

4. Hinz J, Hahn G, Neumann P, Sydow M, Mohrenweiser P, Hellige G, Burchardi H (2003) End-expiratory lung impedance change enables bedside monitoring of end-expiratory lung volume change. Intensive Care Med 29:37-43

5. Erlandsson K, Odenstedt H, Lundin S, Stenqvist O (2006) Positive endexpiratory pressure optimization using electric impedance tomography in morbidly obese patients during laparoscopic gastric bypass surgery. Acta Anaesthesiol Scand 50:833-839

6. Lindgren S, Odenstedt H, Erlandsson K, Grivans C, Lundin S, Stenqvist O (2008) Bronchoscopic suctioning may cause lung collapse: a lung model and clinical evaluation. Acta Anaesthesiol Scand 52:209-218

7. Odenstedt H, Lindgren S, Olegard C, Erlandsson K, Lethvall S, Aneman A, Stenqvist O, Lundin S (2005) Slow moderate pressure recruitment maneuver minimizes negative circulatory and lung mechanic side effects: evaluation of recruitment maneuvers using electric impedance tomography. Intensive Care Med 31:1706-1714
8. Kunst PW, de Vries PM, Postmus PE, Bakker J (1999) Evaluation of electrical impedance tomography in the measurement of PEEP-induced changes in lung volume. Chest 115:1102-1106

9. Schibler A, Calzia E (2008) Electrical impedance tomography: a future item on the "Christmas Wish List" of the intensivist? Intensive Care Med 34:400-401

10. Olegard C, Sondergaard S, Houltz E, Lundin S, Stenqvist O (2005) Estimation of functional residual capacity at the bedside using standard monitoring equipment: a modified nitrogen washout/washin technique requiring a small change of the inspired oxygen fraction. Anesth Analg 101:206-212

11. Chiumello D, Cressoni M, Chierichetti M, Tallarini F, Botticelli M, Berto V, Mietto C, Gattinoni L (2008) Nitrogen washout/ washin, helium dilution and computed tomography in the assessment of end expiratory lung volume. Crit Care 12:R150

12. Luepschen H, Meier T, Grossherr M, Leibecke T, Karsten J, Leonhardt S (2007) Protective ventilation using electrical impedance tomography. Physiol Meas 28:S247-S260

13. Lindgren S, Odenstedt $\mathrm{H}$, Olegard $\mathrm{C}$, Sondergaard S, Lundin S, Stenqvist O (2007) Regional lung derecruitment after endotracheal suction during volume- or pressure-controlled ventilation: a study using electric impedance tomography. Intensive Care Med 33:172-180

14. Pasquali E (1967) Problems in impedance pneumography: electrical characteristics of skin and lung tissue. Med Biol Eng 5:249-258
15. Zhang J, Patterson RP (2005) EIT images of ventilation: what contributes to the resistivity changes? Physiol Meas 26:S81-S92

16. Victorino JA, Borges JB, Okamoto VN, Matos GF, Tucci MR, Caramez MP, Tanaka H, Sipmann FS, Santos DC, Barbas CS, Carvalho CR, Amato MB (2004) Imbalances in regional lung ventilation: a validation study on electrical impedance tomography. Am J Respir Crit Care Med 169:791-800

17. Frerichs I, Hinz J, Herrmann P, Weisser G, Hahn G, Dudykevych T, Quintel M, Hellige G (2002) Detection of local lung air content by electrical impedance tomography compared with electron beam CT. J Appl Physiol 93:660-666

18. Meier T, Luepschen H, Karsten J, Leibecke T, Grossherr M, Gehring H, Leonhardt S (2008) Assessment of regional lung recruitment and derecruitment during a PEEP trial based on electrical impedance tomography. Intensive Care Med 34:543-550 